\title{
Meta-analysis of Seven Randomized Control Trials to Assess the Efficacy and Toxicity of Combining EGFR-TKI with Chemotherapy for Patients with Advanced NSCLC who Failed First-Line Treatment
}

\author{
Bing-Kun Xiao' ${ }^{1}$ Jian-Yun Yang ${ }^{1}$, Jun-Xing Dong ${ }^{1}$, Zhao-Shuai $\mathrm{Ji}^{2}$, Hai-Yan $\mathrm{Si}^{3}$, \\ Wei-Lan Wang ${ }^{4 *}$, Rong-Qing Huang ${ }^{1 *}$
}

\begin{abstract}
Background: Some recent clinical trials have been conducted to evaluate a combination of EGFR- TKI with chemotherapy for advanced NSCLC patients as second-line therapy, but the results on the efficacy of such trials are inconsistent. The aim of this meta-analysis was to evaluate the efficacy and safety of combination of EGFR-TKI and chemotherapy for patients with advanced NSCLC who failed first-line treatment. Materials and Methods: We searched relative trials from PubMed, EMBASE, ASCO Abstracts, ESMO Abstracts, Cochrane Library and Clinical Trials.gov. Outcomes analyzed were overall response rate (ORR), progression- free survival (PFS), overall survival (OS) and major toxicity. Results: Seven trails eventually were included in this meta-analysis, covering 1,168 patients. The results showed that the combined regimen arm had a significant higher ORR (RR $1.76[1.16,2.66], p=0.007)$ and longer PFS (HR 0.75 $[0.66-0.85], p<0.00001)$, but failed to show effects on OS (HR $0.88[0.68-1.15], p=0.36)$. In terms of subgroup results, continuation of EGFR-TKI in addition to chemotherapy after first-line EGFR-TKI resistance confered no improvement in ORR (RR $0.95[0.68,1.33], p=0.75)$ and PFS (HR 0.89[0.69, 1.15], $p=0.38$ ), and OS was even shorter (HR1.52 [1.05- 2.21], $p=0.03$ ). However, combination therapy with EGFR-TKI and chemotherapy after failure of first-line chemotherapy significantly improved the ORR (RR 2.06 [1.42, 2.99], $p=0.0002)$, PFS (HR 0.71 [0.61, 0.82], $p<0.00001)$ and OS (HR 0.74 [0.62- 0.88], $p=0.0008$ ), clinical benefit being restricted to combining EGFR-TKI with pemetrexed, but not docetaxel. Grade 3-4 toxicity was found at significantly higher incidence in the combined regimen arm. Conclusions: Continuation of EGFR-TKI in addition to chemotherapy after first-line EGFR-TKI resistance should be avoided. Combination therapy of EGFR-TKI and pemetrexed for advanced NSCLC should be further investigated for prognostic and predictive factors to find the group with the highest benefit of the combination strategy.
\end{abstract}

Keywords: EGFR-TKI - chemotherapy - non-small cell lung cancer - meta-analysis - RCTs

Asian Pac J Cancer Prev, 16 (7), 2915-2921

\section{Introduction}

Lung cancer is the leading cause of cancer-related death worldwide (Jemal et al., 2010; Song,. et al., 2014). Combination platinum chemotherapy has been shown to improve the survival of patients for advanced non-smallcell lung cancer (NSCLC), but the expected response rate is only approximately 30\% (Schiller et al., 2002). Most patients with advanced NSCLC eventually show tumor progression after standard first-line platinumbased combination chemotherapy (Aydiner et al., 2013). Docetaxel, pemetrexed and EGFR-TKI have been approved for second-line treatment, but only about $10 \%$ of patients respond to monotherapy using any of these agents (Shepherd et al., 2000; Hanna et al., 2004; Kim et al., 2008; Di Maio et al., 2009). EGFR-TKI is a potent tyrosine kinase inhibitor of epidermal growth factor receptor, which is recommended as first-line therapy in patients with advanced NSCLC who have known active sensitizing EGFR mutation (Linardou et al., 2009; Dahabreh et al., 2010).

A recent meta-analysis showed that combination of EGFR-TKIs and chemotherapy leads to progression-free survival (PFS) benefit as first-line treatment for advanced NSCLC, regardless of EGFR-mutation status, but has no demonstrable impact on OS (Yang et al., 2013). However, it did not explore the effect of combination therapy mainly pemetrexed-based therapy plus EGFR-TKI for advanced NSCLC, which showed a strong synergism in NSCLC cells regardless of the presence or absence of sensitizing 
EGFR mutations (Giovannetti et al., 2008).

Some recent clinical trials have been conducted to evaluate combination of EGFR-TKI with chemotherapy for advanced NSCLC patients as second-line therapy, but the results on the efficacy of such trials are inconsistent( Aerts et al., 2013; Halmos et al., 2013; Lee et al., 2013; Li et al., 2013; Auliac et al., 2014; Dittrich et al., 2014; Mok et al., 2014). Therefore, we conducted this metaanalysis to systematically study the efficacy and toxicity of combination of EGFR-TKI and chemotherapy for patients with advanced NSCLC who failed first-line treatment. Subgroup analysis was performed according to different first-line treatment and different chemotherapeutic agents in combination with EGFR-TKI to discuss their potential clinical applications and the better combination strategy.

\section{Materials and Methods}

\section{Search strategy}

We have collected the eligible trials by searching the PubMed, EMBASE, ASCO Abstracts, ESMO Abstracts, Cochrane Library and Clinical Trials.gov for relevant clinical trials up to September 2014. The keywords were used as follow: "erlotinib OR tarceva," "gefitinib OR iressa", "EGFR-TKI," and "non small cell lung cancer OR NSCLC". All the randomized controlled trials on combination of EGFR-TKI and chemotherapy as secondline therapy for NSCLC were collected and identified. We have also searched all reference lists from trials selected by electronic searching to look for trials that may have been overlooked.

\section{Inclusion criteria}

The randomized controlled trials (RCTs) were eligible for inclusion in our meta-analysis if combined regimen of EGFR-TKI and chemotherapy was compared with chemotherapy or EGFR-TKI monotherapy in patients with NSCLC after failure of first-line treatment. Phase I study and phase II study with only one single arm were excluded.All full of papers on original data were included. Abstracts were also included if sufficient information on study design and outcomes was available.

\section{Data extraction and quality assessment}

Two investigators independently identified eligible trials and discrepancies were resolved by an independent expert. The data collected included baseline patient characteristics: first author, year of publication, name and the timing of administration of EGFR-TKI and chemotherapy, number of patients in two arms, sex, age, race, performance status (smoking history and PS). The outcomes investigated included overall response rate (ORR), progression-free survival (PFS), overall survival (OS) and toxicities. Only grade 3 or 4 toxicities were analyzed.

The methodological quality of the studies was assessed by using the Jadad score (Jadad et al., 1996). We graded each parameter of trial quality as "good" when the score is 3 to 5 .
A hazard ratio (HR) and associated $95 \%$ CIs was used for results of comparing PFS and OS in both arms. Relative risk (RR) and its $95 \% \mathrm{CI}$ were calculated to assess ORR and grade 3 or 4 toxicities.

Heterogeneity in the results of the trials was estimated by the chi-square test, and $\mathrm{I}^{2}$ index were chosen accordingly (Higgins et al., 2003). When there was no statistically significant heterogeneity, a pooled effect was calculated with a fixed-effect model. When heterogeneity was observed ( $p<0.1$, or $\left.\mathrm{I}^{2}>50 \%\right)$, further analysis (subgroup analysis or random-effect model) was conducted to identify the potential cause. All reported $p$ values were two-sided and $p$ values less than 0.05 were regarded as statistically significant. All meta-analysis were performed by Review Manager 5.2.

\section{Results}

\section{Characteristics of the included trials}

We reviewed 1639 potentially relevant trials from our initial search strategies. After exclusion of duplicate and irrelevant studies, seven trials were highly eligible for inclusion in this meta-analysis. Of the included studies, two studies compared continuation of EGFRTKI in addition to chemotherapy vs chemotherapy after progression on first-line EGFR-TKI (Halmos et al., 2013; Mok et al., 2014), five studies compared EGFR-TKI plus chemotherapy vs chemotherapy or EGFR-TKI alone after progression on first-line chemotherapy (Aerts et al., 2013; Lee et al., 2013; Li et al., 2013; Auliac et al., 2014; Dittrich et al., 2014). The study search process is shown in a flow chart (Figure 1). Baseline characteristics of the seven trials are provided in Table 1.

Jadad score was used to assess the quality of the included trials. Overall, six studies scored 3 , one scored 5.

\section{Overall response rate}

All the 7 trials reported outcome of ORR. The pooled RR for ORR showed that EGFR-TKI plus chemotherapy as second-line therapy significantly improved the ORR (RR 1.76 [1.16, 2.66], $p=0.007$ ), based on the random effects model, due to significant heterogeneity $\left(\mathrm{I}^{2}=62 \%\right)$. Subgroup analyses were performed according to different first-line therapy. Significant ORR improvement was observed in the combined regimen of TKI and chemotherapy group after progression on first-line chemotherapy (RR 2.06 [1.42, 2.99], $p=0.0002$ ), but not

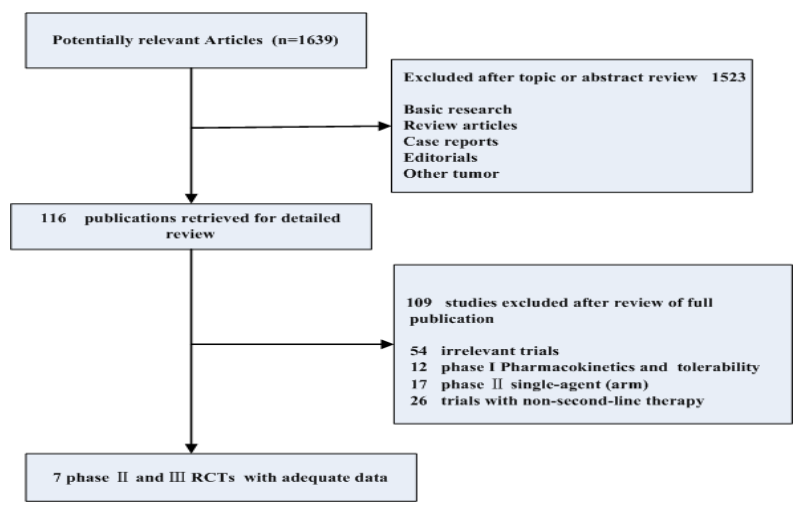

Figure 1. Outline of the Search-flow Diagram 
Meta-analysis of RCTs to Assess the Combining EGFR-TKI with Chemotherapy for Patients with Advanced NSCLC Table 1. Characteristic of the Seven Eligible Randomized Trails in this Meta-analysis

\begin{tabular}{|c|c|c|c|c|c|c|c|c|}
\hline Authors and year & Phase & Regimens(per arm) & $\begin{array}{l}\text { Patients } \\
\text { enrolled }\end{array}$ & $\begin{array}{l}\text { Male } \\
(\%)\end{array}$ & $\begin{array}{l}\text { Median } \\
\text { age }\end{array}$ & $\begin{array}{c}\text { Smoker } \\
(\%)\end{array}$ & $\begin{array}{c}\text { Race (\%) } \\
\text { (Asian) }\end{array}$ & $\begin{array}{c}\text { ECOG } \\
\text { PS 0/1(\%) }\end{array}$ \\
\hline \multirow[t]{2}{*}{ Dittrich et al 2014} & II & $\operatorname{Pem}(\mathrm{d} 1)+\operatorname{Erl}($ daily $), \mathrm{q} 3 \mathrm{w}$ & 76 & $46(61)$ & 64 & 86.8 & $<2 \%$ & $66(88)$ \\
\hline & & $\operatorname{Pem}(d 1), q 3 w$ & 83 & $49(59)$ & 61 & 83.1 & $<2 \%$ & $72(86.8)$ \\
\hline \multirow[t]{3}{*}{ Lee et al 2013} & II & $\operatorname{Pem}(\mathrm{d} 1)+\operatorname{Erl}(\mathrm{d} 2-14), \mathrm{q} 3 \mathrm{w}$ & 78 & $20(26)$ & 55.8 & $0(0)$ & $41(53)$ & $71(91.0)$ \\
\hline & & $\operatorname{Erl(daily)}$ & 82 & $28(34)$ & 53.9 & $0(0)$ & $49(60)$ & $76(92.7)$ \\
\hline & & $\operatorname{Pem}(d 1), q 3 w$ & 80 & $35(44)$ & 55.9 & $0(0)$ & $43(54)$ & $76(95.0)$ \\
\hline \multirow[t]{2}{*}{ Aerts et al 2013} & II & $\mathrm{CT}(\mathrm{d} 1)+\operatorname{Erl}(\mathrm{d} 2-16), \mathrm{q} 3 \mathrm{w} * 4$ & 116 & $73(63)$ & 62.5 & $97(84)$ & NR & $106(91)$ \\
\hline & & Erl(daily) & 115 & $75(65)$ & 64 & $98(85)$ & NR & $106(92)$ \\
\hline \multirow[t]{2}{*}{ Auliac et al 2014} & II & $\operatorname{Doc}(\mathrm{d} 1)+\operatorname{Erl}(\mathrm{d} 2-16), \mathrm{q} 3 \mathrm{w}$ & 73 & $59(81)$ & 59.1 & $63(88)$ & NR & $66(90)$ \\
\hline & & $\operatorname{Doc}(\mathrm{d} 1), \mathrm{q} 3 \mathrm{w}$ & 74 & $56(76)$ & 59.7 & $72(97)$ & NR & $61(82)$ \\
\hline \multirow[t]{2}{*}{ Li et al 2013} & II & $\operatorname{Pem}(\mathrm{d} 1)+\operatorname{Erl}(\mathrm{d} 2-17), \mathrm{q} 3 \mathrm{w}$ & 53 & $24(45)$ & 62 & $36(68)$ & $2(4)$ & $47(89)$ \\
\hline & & $\operatorname{Pem}(d 1), q 3 w$ & 27 & $14(52)$ & 64 & $22(81)$ & $0(0)$ & $24(89)$ \\
\hline \multirow{2}{*}{ Mok et al 2014} & III & Gef(daily)+Pem(d1)+Cis(d1), q3w & 133 & $46(35)$ & 59 & $45(34)$ & NR & $133(100)$ \\
\hline & & $\operatorname{Pem}(\mathrm{d} 1)+\operatorname{Cis}(\mathrm{d} 1), \mathrm{q} 3 \mathrm{w}$ & 132 & $48(36)$ & 57 & $41(31)$ & NR & $132(100)$ \\
\hline \multirow{2}{*}{ Halmos et al 2013} & II & $\mathrm{CT}(\mathrm{d} 1)+\operatorname{Erl}(\mathrm{d} 2-19), \mathrm{q} 3 \mathrm{w}$ & 22 & $10(45)$ & 63.5 & NR & $0(0)$ & NR \\
\hline & & $\mathrm{CT}(\mathrm{d} 1), \mathrm{q} 3 \mathrm{w}$ & 24 & $5(21)$ & 67 & NR & $0(0)$ & NR \\
\hline
\end{tabular}

*ECOG PS, Eastern Cooperative Oncology Group performance status. Erl: Erlotinib. Gef: Gefitinib Car: Carboplatin. Docetaxel: Doc. Pem:Pemetrexed. Cis: Cisplatin. CT: Chemotherapy. NR: No Report

improvement in continuation of EGFR-TKI addition to chemotherapy group after progression on first-line TKI (RR 0.95 [0.68, 1.33], $p=0.75$ ) (Figure 2).

\section{Progression-free survival (PFS)}

All 7 trials included in the analysis provided PFS results. Compared to chemotherapy or EGFR-TKI alone, the combination of TKI and chemotherapy resulted in statistically significant improvement in PFS (HR 0.75 [0.66-0.85], $p<0.00001)$, without apparent heterogeneity among the studies $\left(p=0.11, \mathrm{I}^{2}=40 \%\right)$.

Subgroup analysis showed that the beneficial effect was restricted to the combined regimen of TKI and chemotherapy arm after progression on first-line chemotherapy (HR 0.71 [0.61,0.82], $p<0.00001$ ), whereas continuation of EGFR-TKI addition to chemotherapy arm after progression on first-line TKI did not show a significant difference compared with TKI or chemotherapy alone (HR 0.89 [0.69, 1.15], $p=0.38$ ) (Figure 3).

In addition, we took further subgroup analyses in patients with advanced NSCLC who failed first-line chemotherapy to define potentially benefit from different chemotherapeutic agents in combination with EGFRTKI(Aerts et al., 2013; Lee et al., 2013; Li et al., 2013; Auliac et al., 2014; Dittrich et al., 2014). Results showed that PFS improvement was only observed in combining pemetrexed with EGFR-TKI group (HR 0.63 [0.53,0.74], $p<0.00001)$, whereas docetaxel with EGFR-TKI group did not show significant improvement in PFS (HR 1.01 [0.77, 1.34], $p=0.92$ ) (Figure 4).

\section{Overall survival (OS)}

Just 6 trials on the data of OS were available. The trial by Auliac et al. didn't give the HRs, associated 95\%CIs or survival curves for OS (Auliac et al., 2014). There was no significant difference between the combined regimen arm and monotherapy arm (HR 0.88 [0.68-1.15], $p=0.36$ ). Nevertheless, there might be substantial heterogeneity in the HRs for OS from the individual trials $\left(p=0.02, \mathrm{I}^{2}=61 \%\right)$ and we incorporated it into random-effects model.

Results were inconsistent when subgroup analyses

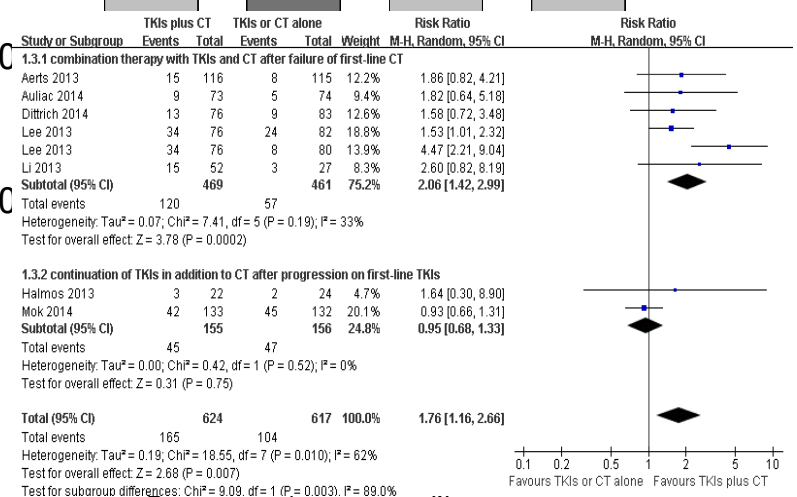

Figure 2. Comparison TKIs plus CT with TKIs or CT Alone as Second-line Therapy in ORR (random-effects model). TKIs; Tytosine kinase inhibitors, CT; Chemotherapy

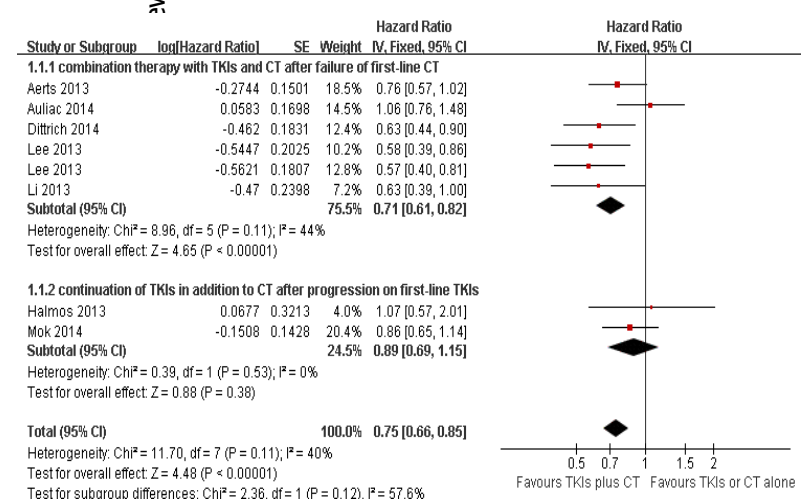

Figure 3. Comparison TKIs Plus CT with TKIs or CT alone as Second-line Therapy in PFS (fixed-effect model). TKIs; Tytosine kinase inhibitors, CT; Chemotherapy

were conducted. Significantly OS benefit was observed from chemotherapy alone arm after progression on firstline TKI (HR1.52 [1.05-2.21], $p=0.03$ ), whereas OS benefit was observed from the combined regimen arm after progression on first-line chemotherapy (HR 0.74 [0.62-0.88], $p=0.0008$ ) (Figure 5). And in further subgroup analysis for patients with advanced NSCLC who failed first-line chemotherapy(Aerts et al., 2013; Lee et al., 2013; Li et al., 2013; Dittrich et al., 2014), the OS benefit was again restricted to combining pemetrexed with EGFR-TKI 


\section{Toxicities}

This meta-analysis assessed the toxicities with grade 3 or 4 toxicities of EGFR-TKI with chemotherapy vs. EGFR-TKI or chemotherapy group. The analysis showed the combined regimen caused more grade 3 or 4 anemia (RR $1.73[1.11,2.69], p=0.01)\left(p=0.09, \mathrm{I}^{2}\right.$ $=45 \%)$, leukopenia (RR 3.51 [2.25, 5.50], $p<0.00001)$ $\left(p=0.18, \mathrm{I}^{2}=34 \%\right)$, neutropenia (RR 1.79 [1.32, 2.42], $p=0.0002)\left(p=0.04, \mathrm{I}^{2}=55 \%\right)$, thrombocytopenia (RR 2.59 [1.12, 6.00], $p=0.03)\left(p=0.27, \mathrm{I}^{2}=24 \%\right)$, rash $(\mathrm{RR}$ $2.78[1.55,4.98], p=0.0006)\left(p=0.32, \mathrm{I}^{2}=15 \%\right)$, diarrhoea

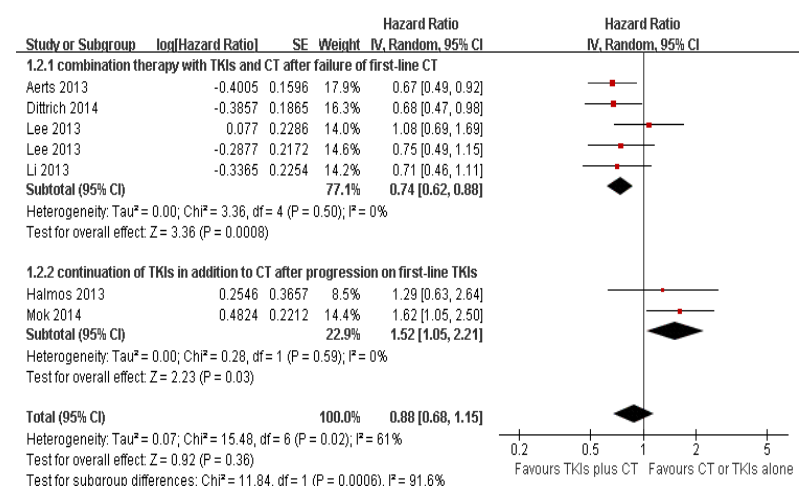

Figure 4. Comparison TKIs plus CT with TKIs or CT alone as second-line therapy in OS (random-effects model). TKIs; Tytosine kinase inhibitors, CT; Chemotherapy

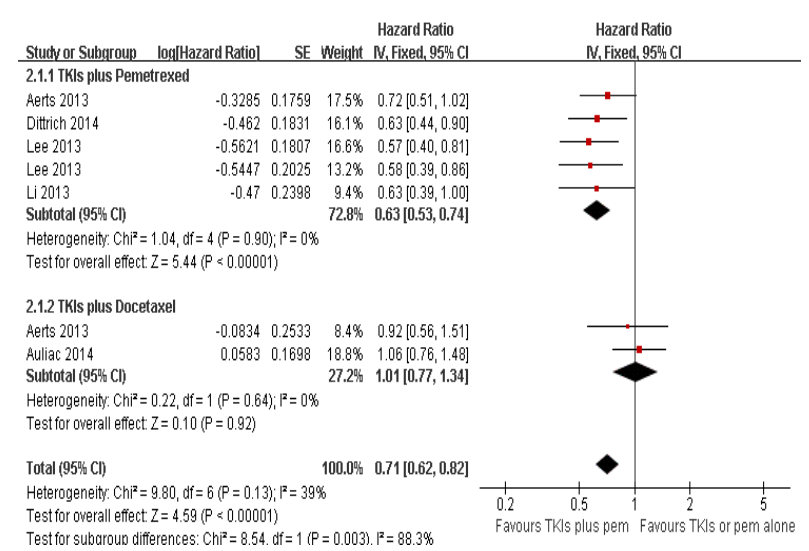

Figure 5. Comparison TKIs plus CT with TKIs or CT alone after failure of first-line CT in PFS (fixed-effect model). TKIs; Tytosine kinase inhibitors, CT; Chemotherapy

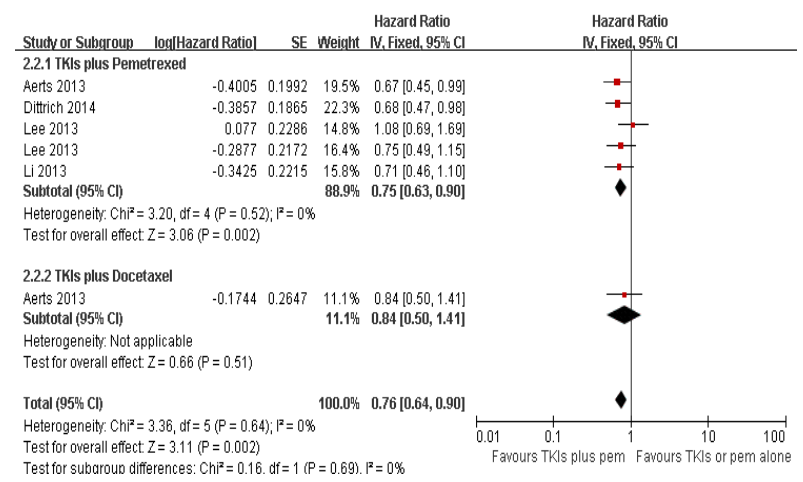

Figure 6. Comparison TKIs plus CT with TKIs or CT alone after failure of first-line $\mathrm{CT}$ in OS (fixed-effect model). TKIs; Tytosine kinase inhibitors, CT; Chemotherapy

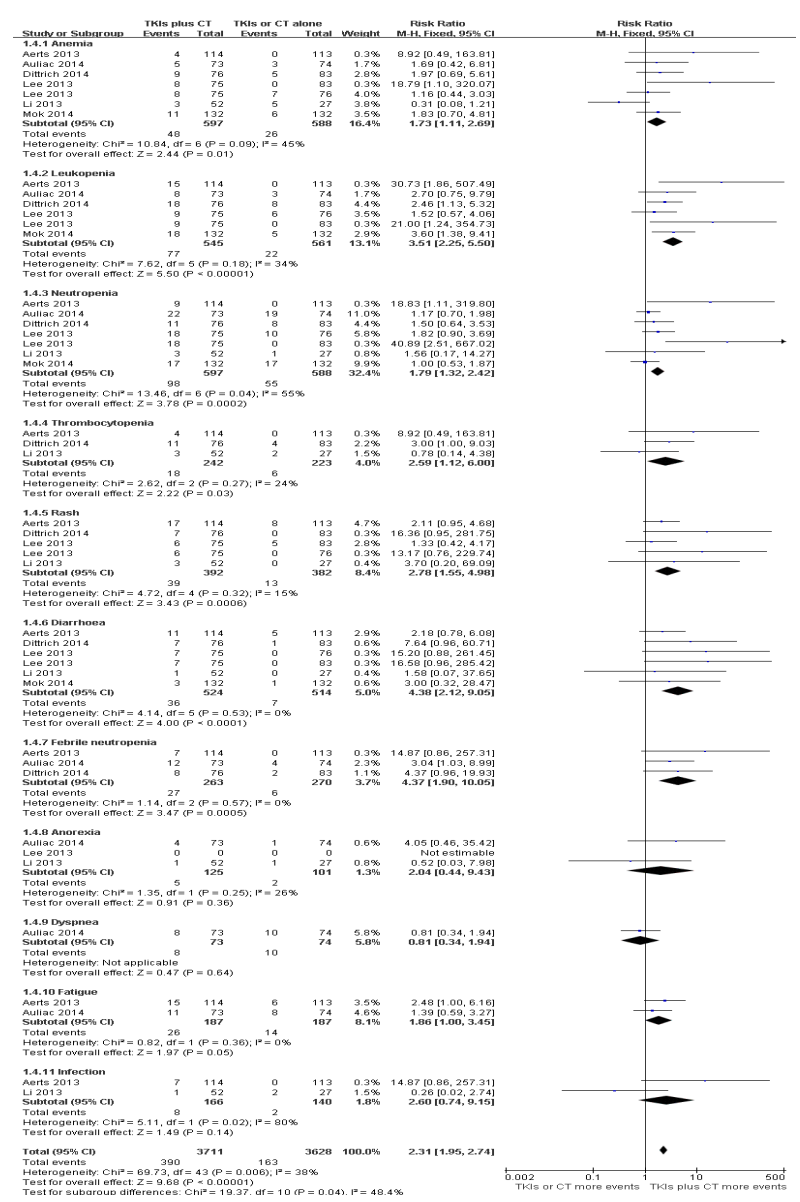

Figure 7. Comparison of grade 3 or 4 toxicities between TKIs plus CT and TKIs or CT alone. TKIs; Tytosine kinase inhibitors, CT; Chemotherapy

$(\operatorname{RR} 4.38[2.12,9.05], p<0.0001)\left(p=0.53, \mathrm{I}^{2}=0 \%\right)$, febrile neutropenia(RR 4.37[1.90, 10.05], $p=0.0005)(p=0.57$, $\mathrm{I}^{2}=0 \%$ ). And there were no differences of other severe toxicities between the two arms (Figure 7).

\section{Publication bias}

To minimize the publication bias, we selected papers strictly according to inclusion criteria. Furthermore, publication bias was detected by funnel plot. No apparent publication bias was found in the analysis.

\section{Discussion}

About 40-50\% NSCLC patients will receive secondline therapy after failure of first-line standard therapy (Stinchcombe and Socinski, 2009). However, available for second-line treatment options are limited: docetaxel, pemetrexed, and erlotinib (Massarelli and Herbst, 2006). Any second-line treatment should represent a rational selection of a drug or drug combination depending on their activity against tumors pretreated with distinct firstline therapies (Gandara et al., 2009). The combination of pemetrexed or docetaxel and EGFR-TKI has demonstrated synergistic or additive activity in preclinical studies.

However, the results on the efficacy of several RCTs conducted to evaluate combination of chemotherapy and EGFR-TKI as second-line therapy for advanced NSCLC are inconsistent. The IMPRESS study reported 
at ESMO 2014 congress showed that continuation of gefitinib in addition to pemetrexed/cisplatin would be of no clinical benefit for patients with NSCLC after progression on first-line gefitinib (Mok et al., 2014). And a phase II trial also showed that no benefit was seen with the continuation of erlotinib in addition to chemotherapy (docetaxel or pemetrexed) as compared to chemotherapy (docetaxel or pemetrexed) alone in patients who had previously benefited from erlotinib but then showed progression(Halmos et al., 2013). For patients with advanced NSCLC who failed first-line chemotherapy, combination of docetaxel and erlotinib as second-line was found not more effective than monotherapy in two phase II randomized trials (Aerts et al., 2013; Auliac et al., 2014). Nevertheless, combination of pemetrexed and erlotinib as second-line in four RCTs showed promising clinical activity after failure of first-line chemotherapy (Aerts et al., 2013; Lee et al., 2013; Li et al., 2013; Dittrich et al., 2014), regardless of whether concomitant or sequential combination, unselected or enriched patients. As a result, it is of particular importance to determine whether combination of EGFR-TKI and chemotherapy after failure of first-line treatment would increase clinical activity. The results of our meta-analysis showed that the combined regimen arm resulted in a significant higher ORR (RR $1.76[1.16,2.66], p=0.007)$ and longer PFS (HR0.75 [0.66$0.85], p<0.00001)$, but failed to show OS improvement (HR0.88 [0.68-1.15], $p=0.36$ ). Subgroup analysis showed that different first-line therapy resulted in different clinical effect of combination of EGFR-TKI and chemotherapy as second-line therapy. Continuation of EGFR-TKI in addition to chemotherapy at the time of EGFR-TKI resistance which was formerly chosen by many clinicians was confirmed no improvement in ORR, PFS and OS. Several factors may contribute to the negative study results, including a possible antagonism between platinum and EGFR-TKI regardless of the EGFR mutation status (Tsai et al., 2011; Goldberg et al., 2013; Tsai et al., 2013), and no enhanced antitumor effect on the growth of TKIresistant NSCLC cells with the T790M mutation (about $50 \%$ acquisition of EGFR) for combination of EGFRTKI and pemetrexed therapy (Takezawa et al., 2010). Of course, there are other mechanisms of resistance to EGFR TKIs, including MET amplification, HGF overexpression, PIK3CA mutations and PDGFR expression et al (Remon et al., 2014). Dynamics of resistance evolution and the question of heterogeneity add to complexity of the problem. However, we found that combination therapy with EGFR-TKI and chemotherapy after failure of firstline chemotherapy significantly improved the ORR (RR 2.06 [1.42, 2.99], $p=0.0002$ ), PFS (HR 0.71 [0.61, 0.82], $p<0.00001)$ and OS (HR 0.74 [0.62-0.88], $p=0.0008$ ), which clinical benefit was restricted to combining pemetrexed with EGFR-TKI. Many preclinical studies showed that EGFR-TKI and pemetrexed had a strong synergism in NSCLC cells, regardless of the presence or absence of sensitizing EGFR mutations. Pemetrexed may increase EGFR phosphorylation and reduce Akt phosphorylation (sensitizing tumor cells to erlotinib), while erlotinib was found to reduce thymidylate synthase expression and activity, which in turn may sensitize tumor cells to pemetrexed (Giovannetti et al., 2008). In addition, non cisplatin-based double chemotherapy in these trials may be another reason to result in clinical effect. Nevertheless, it is difficult to explain why our meta-analysis showed that combination docetaxel with EGFR-TKI after failure of first-line chemotherapy did not improvement PFS (HR 1.01 [0.77, 1.34], $p=0.92$ ) and OS (HR $0.84[0.50,1.41], p=0.51$ ), because combined EGFR-TKI and antimicrotubule agents showed synergism in NSCLC cells harboring no sensitizing EGFR mutations in many preclinal studies (Mahaffey et al., 2007; Tsai et al., 2012). The same negative results of combined EGFR-TKI and antimicrotubule agents as first-line therapy were found in TRIBUTE study (Herbst et al., 2005), INTACT -2 trial (Herbst et al., 2004), CALGB 30406 trial (Janne et al., 2012) and a randomized phase II by Hirsh et al (Hirsch et al., 2011), regardless of histology type, administration sequence, EGFR mutations status, whether patients was unselected or enriched population, and whether combining platinum or not. Thus we consider that combination antimicrotubule agents with EGFR-TKI may be not a good strategy for advanced NSCLC.

As expected, the combination produced more toxicity than either single agent. Grade 3 or 4 toxicities increased with combination therapy were anemia, leukopenia, neutropenia, thrombocytopenia, rash, diarrhea and febrile neutropenia. However, these side effects were generally clinically manageable.

There are several limitations in this meta-analysis. First, although the publication bias was not found by funnel plots, the small number of phase III trial and the small number of patients for inclusion limited the power of the analysis. Second, we did not perform stratification analysis for the EGFR mutations, regimen in control group or administration sequence due to small sample size or absence of original data. Third, heterogeneity among trials was found in our meta-analysis. We applied a random effect model that takes possible heterogeneity into consideration.

In conclusion, our meta-analysis showed that different first-line therapy resulted in different clinical effect of combination of EGFR-TKI and chemotherapy as secondline therapy. Continuation of EGFR-TKI in addition to chemotherapy at the time of EGFR-TKI resistance should be avoided. Combination therapy with EGFRTKI and pemetrexed for advanced NSCLC showed better activity and should be further investigated prognostic and predictive factors to find the group with the highest benefit of the combination.

\section{Acknowledgements}

The study was supported by Beijing Natural Science Foundation (7142125). The authors declare that they have no competing interests.

\section{References}

Aerts JG, Codrington H, Lankheet NA, et al (2013). A randomized phase II study comparing erlotinib versus erlotinib with alternating chemotherapy in relapsed non- 
small-cell lung cancer patients: the NVALT-10 study. Ann Oncol, 24, 2860-5.

Auliac JB, Chouaid C, Greiller L, et al (2014). Randomized open-label non-comparative multicenter phase II trial of sequential erlotinib and docetaxel versus docetaxel alone in patients with non-small-cell lung cancer after failure of first-line chemotherapy: GFPC 10.02 study. Lung Cancer, 85, 415-19.

Aydiner A, Yildiz I, Seyidova A (2013). Clinical outcomes and prognostic factors associated with the response to erlotinib in non-small cell lung cancer patients with unknown EGFR mutational status. Asian Pac J Cancer Prev, 14, 3255-61.

Dahabreh IJ,Linardou H, Siannis F, et al (2010).Somatic EGFR mutation and gene copy gain as predictive biomarkers for response to tyrosine kinase inhibitors in non-small cell lung cancer. Clin Cancer Res, 16, 291-303.

Di Maio M, Chiodini P, Georgoulias V, et al (2009). Metaanalysis of single-agent chemotherapy compared with combination chemotherapy as second-line treatment of advanced non-small cell lung cancer. J Clin Oncol, 27, 1836-43.

Dittrich C, Papai-Szekely Z, Vinolas N, et al (2014).A randomized phase II study of pemetrexed versus pemetrexed+erlotinib as second-line treatment for locally advanced or metastatic non-squamous non-small cell lung cancer. Eur J Cancer, 50, 1571-80.

Gandara DR, Mack PC, Li T, Lara PN Jr, Herbst RS (2009). Evolving treatment algorithms for advanced non-small-cell lung cancer: 2009 looking towards 2012. Clin Lung Cancer, 10, 392-4.

Giovannetti E, Lemos C, Tekle C, et al (2008). Molecular mechanisms underlying the synergistic interaction of erlotinib, an epidermal growth factor receptor tyrosine kinase inhibitor, with the multitargeted antifolate pemetrexed in non-small-cell lung cancer cells. Mol Pharmacol, 73, 1290-300.

Goldberg SB, Oxnard GR, Digumarthy S, et al (2013). Chemotherapy with erlotinib or chemotherapy alone in advanced non-small cell lung cancer with acquired resistance to EGFR tyrosine kinase inhibitors. Oncologist, 18, 1214-20.

Halmos B, Pennell NA, Otterson GA, et al (2013). Erlotinib beyond progression study: Randomized phase II study comparing chemotherapy plus erlotinib with chemotherapy alone in EGFR TKI-responsive, advanced non-small cell lung cancer (NSCLC) that subsequently progresses. J Clin Oncol, 31, (suppl; abstr 8114).

Hanna N, Shepherd F, Fossella F, et al (2004). Randomized phase III trial of pemetrexed versus docetaxel in patients with non-small-cell lung cancer previously treated with chemotherapy. J Clin Oncol, 22, 1589-97.

Herbst RS, Giaccone G, Schiller JH, et al (2004). Gefitinib in combination with paclitaxel and carboplatin in advanced non-small-cell lung cancer: a phase III trial-INTACT 2. $J$ Clin Oncol, 22, 785-94.

Herbst RS, Prager D, Hermann R, et al (2005). TRIBUTE: a phase III trial of erlotinib hydrochloride (OSI-774) combined with carboplatin and paclitaxel chemotherapy in advanced non-small-cell lung cancer. J Clin Oncol, 23, 5892-9.

Higgins JP, Thompson SG, Deeks JJ, et al (2003). Measuring inconsistency in meta-analyses. BJM, 327, 557-60.

Hirsch FR, Kabbinavar F, Eisen T, et al (2011). A randomized, phase II, biomarker-selected study comparing erlotinib to erlotinib intercalated with chemotherapy in first-line therapy for advanced non-small-cell lung cancer. J Clin Oncol, 29, 3567-73.

Jadad AR, Moore RA, Carroll D, et al (1996). Assessing the quality of reports of randomized clinical trials: Is blinding necessary? Control Clin Trials, 17, 1-12.

Janne PA, Wang X, Socinski MA, et al (2012). Randomized phase II trial of erlotinib alone or with carboplatin and paclitaxel in patients who were never or light former smokers with advanced lung adenocarcinoma: CALGB 30406 trial. $J$ Clin Oncol, 30, 2063-9.

Jemal A, Siegel R, Xu J, Ward E (2010). Cancer statistics, 2010. CA Cancer J Clin, 60, 277-300.

Kim ES, Hirsh V, Mok T, et al (2008). Gefitinib versus docetaxel in previously treated on-small-cell lung cancer (INTEREST): a randomized phase III trial. Lancet, 372, 809-18.

Lee DH, Lee JS, Kim SW, et al (2013). Three-arm randomized controlled phase 2 study comparing pemetrexed and erlotinib to as second-line treatment for never-smokers with nonsquamous non-small cell lung cancer. Eur J Cancer, 49, 3111-21.

Linardou H, Dahabreh IJ, Bafaloukos D, Kosmidis P, Murray S (2009). Somatic EGFR mutations and efficacy of tyrosine kinase inhibitors in NSCLC. Nat Rev Clin Oncol, 6, 352-66.

Li TH, Piperdi B, Walsh WV, et al (2013). Randomized phase II study of pharmacodynamic separation (PDS) of pemetrexed (Pem) and erlotinib (Erl) versus pem alone in patients (pts) with advanced non-small cell lung cancer (NSCLC). J Clin Oncol, 31, 8097.

Mahaffey CM, Davies AM, Lara PN Jr, et al (2007). Scheduledependent apoptosis in K-ras mutant non-small-cell lung cancer cell lines treated with docetaxel and erlotinib: rationale for pharmacodynamic separation. Clin Lung Cancer, 8, 548-53.

Massarelli E, Herbst RS (2006). Use of novel second-line targeted therapies in non-small cell lung cancer. Semin Oncol, 33, 9-16.

Mok TS, Wu YL, Nakagawa K, et al (2014). Gefitinib/ chemotherapy vs chemotherapy in epidermal growth factor receptor (EGFR) mutation-positive non-small-cell lung cancer (NSCLC) after progression on first-line gefitinib: the Phase III, randomised IMPRESS study. Abstract \#LBA2_PR presented at the European Society for Medical Oncology (ESMO) 2014 Congress. Available: http://www. esmo.org. Accessed 26-30 September 2014.

OuYang PY, Su Z, Mao YP, Deng W, Xie FY (2013). Combination of EGFR-TKIs and chemotherapy as first-line therapy for advanced NSCLC: a meta-analysis. PLoS One, 13, 79000.

Remon J, Morán T, Majem M, et al (2014). Acquired resistance to epidermal growth factor receptor tyrosine kinase inhibitors in EGFR-mutant non-small cell lung cancer: a new era begins. Cancer Treat Rev, 40, 93-101.

Schiller JH, Harrington D, Belani CP, et al (2002). Comparison of four chemotherapy regimens for advanced non-small-cell lung cancer. N Engl J Med, 346, 92-8.

Shepherd FA, Dancey J, Ramlau R, et al (2000). Prospective randomized trial of docetaxel versus best supportive care in patients with non-small-cell lung cancer previously treated with platinum-based chemotherapy. J Clin Oncol, 18, 2095-103.

Song T, Yu W, Wu SX (2014). Subsequent treatment choices with acquired resistance to EGFR-TKIs in non-small cell lung cancer: restore after a drug holiday or switch to another EGFR-TKI? Asian Pac J Cancer Prev, 15, 205-13.

Stinchcombe TE, Socinski MA (2009). Current treatments for advanced stage non-small cell lung cancer. Proc Am Thorac Soc, 6, 233-41.

Takezawa K, Okamoto I, Tanizaki J, et al (2010). Enhanced anticancer effect of the combination of BIBW2992 and thymidylate synthase-targeted agents in non-small cell lung cancer with the T790M mutation of epidermal growth factor 

receptor. Mol Cancer Ther, 9, 1647-56.

Tsai CM, Chen JT, Chiu CH, et al (2013). Combined epidermal growth factor receptor (EGFR)-tyrosine kinase inhibitor and chemotherapy in non-small-cell lung cancer: chemorefractoriness of cells harboringsensitizing-EGFR mutations in the presence of gefitinib. Lung Cancer, 82, 305-12.

Tsai CM, Chen JT, Stewart DJ, et al (2011). Antagonism between gefitinib and cisplatin in non-small cell lung cancer cells: why randomized trials failed? J Thorac Oncol, 6, 559-68.

Tsai CM, Chiu CH, Chang KT, et al (2012). Gefitinib enhances cytotoxicities of antimicrotubule agents in non-small-cell lung cancer cells exhibiting no sensitizing epidermal growth factor receptor mutation. J Thorac Oncol, 7, 1218-27. 